\title{
Cumplimiento de las normas legales en el concurso de méritos y oposición del sistema educativo ecuatoriano
}

\section{Compliance with legal rules in the merits contest and opposition of the ecuadorian educational system}

Luis David Velasco Almachi. ${ }^{1}$, Estefanía del Rosario Ortega Araus. ${ }^{2}$, Raúl Yungán Yungán. ${ }^{3}$ \& Verónica Janeth Tenelema Toapanta. ${ }^{4}$

\begin{abstract}
.
DOI: https://doi.org/10.33262/cienciadigital.v4i2.1212

This article includes the process of the contest of merits and opposition in which professionals in education from all over the country participate, to obtain a destination in the public teaching through the program of the Ministry of Education affected "I want to be a teacher". Systematization is manifested to obtain eligibility divided into a merit phase and an opposition phase supported by current regulations and the Information System oef the Ministry of Education (SIME). The modality is bibliographic and field, the purpose of this research is to identify compliance with legal regulations. This is indicated by the Organic Law on Intercultural Education (LOEI) in the second chapter of the merits and opposition contest to fill vacancies for public teachers, in order to see the development and transparency of the process. For this purpose, a self-assessment questionnaire was designed that includes 10 questions, 7 of which are open questions; and 3 questions of alternatives with a section to detail the selected option. This questionnaire was applied to 10 teachers from various institutions that participate or participate in the contest. From the results there are 3 profiles (high, medium and low) in knowledge about the application of legal regulations. The results

\footnotetext{
${ }^{1}$ Universidad Técnica de Ambato, Facultad de Ciencia Humanas y de la Educación, lvelasco4836@uta.edu.ec

${ }^{2}$ Universidad Técnica de Ambato, Facultad de Ciencia Humanas y de la Educación, eortega315@uta.edu.ec,

${ }^{3}$ Universidad Técnica de Ambato, Facultad de Ciencia Humanas y de la Educación, ryungan@uta.edu.ec

${ }^{4}$ Universidad Técnica de Ambato, Facultad de Ciencia Humanas y de la Educación, vtenelema0788@uta.edu.ec
} 
that the investigation shows are the transparency of the process, which takes a long time to complete its phases according to the established schedule and also the ignorance of the bonuses

Keywords: Contests, Merits, Opposition And rules.

\section{Resumen.}

El presente artículo comprende el proceso del concurso de méritos y oposición en los que participan los profesionales en educación de todo el país, para la obtención de un nombramiento en el magisterio público mediante el programa del Ministerio de Educación denominada "Quiero ser maestro". Se manifiesta la sistematización para obtener la elegibilidad dividida en una fase de méritos y una fase de oposición apoyada en la normativa vigente y en el Sistema de Información del Ministerio de Educación (SIME). La modalidad es bibliográfica y de campo, la finalidad de la presente investigación es identificar el cumplimiento de la normativa legal. Así indica la Ley Orgánica de Educación Intercultural (LOEI) en el capítulo segundo del concurso de méritos y oposición para llenar vacantes de docentes públicos, a fin de ver el desarrollo y transparencia del proceso. Con este propósito se diseñó un cuestionario de autoevaluación que incluye 10 preguntas 7 de ellas son pregunta abiertas; y 3 preguntas de alternativas con un apartado para detallar la opción seleccionada. Este cuestionario se aplicó a 10 docentes de varias instituciones quienes participan o participaron en el concurso. A partir de los resultados se encuentran 3 perfiles (alto, medio y bajo) en conocimiento sobre la aplicación de la normativa legal. Los resultados que muestra la investigación es la transparencia del proceso, que este tarda mucho tiempo para completar sus fases según el cronograma establecido y además el desconocimiento de las bonificaciones.

\section{Palabras claves: Concurso, Méritos, Oposición y Normas}

\section{Introducción.}

A nivel educativo es imprescindible reconocer los méritos alcanzados por los profesionales en educación. Además, verificar si se cumplen las normativas legales al momento de llenar vacantes dentro de las instituciones educativas mediante los concursos de méritos y oposición. Por lo tanto, la presente investigación se realiza con el interés de indagar la transparencia del proceso que realiza el Ministerio de Educación, encargado de elegir docentes que ingresaran al magisterio o carrera educativa pública. El impacto que causará dentro de la sociedad será positivo, se dará a conocer el proceso y que se necesita para ser parte del personal docente a nombramiento de una institución educativa. A continuación, se mostrará criterios de autores nacionales e internacionales, entre las cuales citamos las más relevantes para esta investigación: 
La investigación realizada por Jiménez Vanessa sobre el sistema de oposición para poder ingresar a cargos de administración pública en el Ecuador. Se muestra la discusión en torno a los requisitos y la procedencia de los aspirantes a llenar las vacantes, así Jimenez (2005) manifiesta que, para nombrar funcionarios interinos será condición inexcusable que no sea posible, con la urgencia exigida por las circunstancias, la prestación del servicio por funcionarios de Carrera, debiendo justificarse estos extremos ante la Comisión Superior de Personal. El nombramiento deberá recaer en personas que reúnan las condiciones exigidas para el ingreso en el cuerpo a que pertenezca el puesto de trabajo.

Algunos autores también consideran que la oposición no presupone una radical exclusión de los méritos previamente adquiridos por los candidatos. En este sentido, Badillo (2009) afirma que en, "la oposición pura lo que ocurre es que los méritos son examinados y valorados en conjunto con las demás pruebas, sin distinción de fases, como ocurre en el concurso y oposición". Por lo tanto, para Badillo se perfila como necesaria la forma en que la evaluación de méritos se produce y no el hecho mismo de su existencia.

Dentro del ámbito escolar y la organización de la misma, el Ecuador ha palpado un sinnúmero de cambios y variaciones en cuanto a la distribución educativa. Se puede señalar que el enfoque de la presente investigación cuenta con las modificaciones legislativas en torno a las Instituciones de Educación Superior. La investigación permite identificar algunas falencias académicas en cuanto a la bibliografía científica al tomar medidas organizativas en la Educación. (Ganga, F. 2017)

El abuso de la discrecionalidad en las aplicaciones de las acciones afirmativas presentes en los concursos de méritos y oposición, vulnera el principio de igualdad es la contradicción legal analizada. Mediante la Constitución de la Republica y la Ley Orgánica de Servicio Público (LOSEP) se pudo observar las 3 posibles soluciones que se dan a este tipo de vulneración y errónea aplicación de principios. Se vulnera el principio de igualdad con las acciones afirmativas en el objetivo de cumplir con la igualdad real, es decir que todos somos iguales ante la ley. Sin embargo, el trato por separado para estos grupos, vulnera los derechos de los demás. Sobre todo, aquellos que no se encuentran en situación de desigualdad ya que los parámetros de objetividad se ven prácticamente son concretos y valoran su capacidad cognoscitiva. Siendo estos ignorados al proporcionar puntos extra solo por condiciones fuera de lugar, la discriminación y el trato separatista se mantiene vigente.

La calificación en los concursos de méritos y oposición debe hacerse con parámetros objetivos y en ningún caso las autoridades nominadoras podrán intervenir de manera directa o subjetiva y hacer uso de mecanismos discrecionales. Este tipo de irregularidades invalidaran los procesos de selección de personal. Como puede entenderse, inclusive se deja claro que, siendo el concurso de méritos y oposición el mecanismo técnico para determinar la idoneidad de una persona. La selección de personal no puede basarse en apreciaciones particulares mucho menos subjetivas, sino en un sistema de calificaciones, que censuré por cuenta propia cualquier forma de discriminación. (Costa Jaramillo, 2012) 
El campo laboral de un servidor público está inmerso a mucha presión laboral que consiste en la coerción y el acumulamiento de trabajo. Se debe tomar en cuenta que los beneficios económicos son los más importantes, sino el tener estabilidad laboral, a más de ello se debe tener vocación por el servicio público. La vocación consiste en sentirse llamado para desempeñar una actividad o profesión dando lo mejor de sí mismo sin la necesidad de cumplir órdenes que bajan la autoestima del empleado. Un servidor público que ha obtenido un puesto por medio del concurso de méritos y oposiciones implica conducirse con integridad, imparcialidad y honestidad garantizando el uso apropiado de la palabra. Los miembros del servicio público deben poseer la capacidad de servir al resto. Los principios que sustentan las normas para la gestión de los procesos de selección de personal, incidiendo en el concurso público de méritos. (Cari, 2016)

Los requisitos para acceder al concurso es obtener experiencia laboral pero no solamente es la única exigencia, sino que también se necesita la titulación de cuarto nivel y es justo ahí donde viene el problema. Muchos de los que postulan para estos cargos son jóvenes recién graduados de la universidad, que desean obtener una carga laboral pero como hacerlo si no poseen experiencia laboral. La experiencia es una pieza clave para el mismo. Se puede señalar que estamos inmersos en una cadena de desempleo, las fuentes laborales colapsan existen como cien postulantes solo para dos o tres vacantes, pero que podemos hacer frente a ello si lo que prevalece siempre son las amistades y una vez más gana lo ilegal en este tipo de concursos. (García Chávez, 2009)

Consecuencia de lo indicado, y en una suerte de "abuso del derecho", varias personas que han postulado en concursos de oposición y méritos ofertados a nivel nacional por varias instituciones públicas, han considerado autodefinirse en su condición étnica, como pertenecientes a uno de los grupos históricamente vulnerados, sin el sustento o justificación necesaria de efectivamente pertenecer o tener vínculos suficientes para ser considerados como tal. Inclusive son beneficiados de la aplicación de medidas afirmativas por dicha auto definición, lo cual, sin duda alguna y en muchos casos ha generado una distorsión injusta de lo prescrito en la Constitución. (Samaniego Dumas, 2017)

Los concursos de méritos y oposición en su abreviatura son la manera más apropiada de poder ingresar a ocupar un cargo público. Los aspirantes pueden participar para cualquier vacante de su preferencia para poder cubrirla. Dichos concursos constan de varias etapas en la cual el postulante deberá permanecer atento acerca de los cambios que puedan ocurrir a lo largo de este. Por ello los concursos de méritos y oposición se rigen a diferentes lineamientos generales, inclusive las diferentes fases del proceso que conlleva el concurso, así mismo como para dar a conocer aspectos relevantes de valoración y puntaciones respectivas. (Sarango, 2016). Es así que los concursos de méritos y oposición deben ser llevados con transparencia, con justicia y sin discriminación para que los postulantes estén al tanto del procedimiento que se lleva. Sobre todo, en la calificación de las evaluaciones y de esta manera prevenir la corrupción, de cualquier forma, la transparencia nos establece una relación de confianza entre las personas que realizan los concursos y los postulantes. La transparencia y la equidad en los concursos son de suma importancia porque al momento de 
publicar los resultados y nombrar al ganador de la vacante podemos determinar si realmente el proceso fue claro, objetivo y si no se manipulo los resultados.

El proceso de selección es el ente encargado de garantizar que la selección del personal para formar parte de los cargos públicos, deben garantizar los principios de libre concurrencia e igualdad de oportunidades. La concurrencia a los concursos de selección de personas es libre y todas las personas gozarán de las mismas oportunidades y estarán en igualdad de condiciones; la transparencia es otro de los principios que prevalece en esta legislación en cuanto a los procesos de selección de personal. Así mismo sin dejar pasar por alto la garantía de imparcialidad de los órganos encargados de selección del personal que deben llevar a cabo los procedimientos más idóneos para el efecto o el cargo a ocupar. (Asanza, 2018)

La investigación va acorde con el sistema que se encuentra bajo un enfoque parametrizable que define la ejecución del proceso selectivo, de acuerdo a la necesidades propias y requeridas por cada Institución de Educación Superior. El estudio realizado demuestra que el proceso selectivo para personal docente en las Universidades del país es necesario que sea por medio de dichos concursos, los cuales deben tener un sistema que evite la filtración de información. Se considera que dicho concurso es de vital importancia para el desarrollo y progreso de empleos en el Ecuador, creando así grandes plazas de trabajo para los servidores públicos. Se debe motivar a los docentes a que accedan a este tipo de concursos, que permiten el progreso del país como un país intercultural y multiétnico.

El concurso de méritos y oposición es la consecuencia de la planificación del talento humano y debería ser estructurado de acuerdo a las necesidades y capacidad técnica y financiera de la institución. Sobre todo, garantizando en todo momento los criterios de igualdad de trato sin discriminación alguna de los deberes y derechos de las y los postulantes. (Andrea, 2016)

Las necesidades que se presentan dentro del sistema educativo en el Ecuador son varias, es decir existen vacantes por llenar. Se puede señalar que muchas de estas son cubiertas a tiempo porque cada vez se van incrementando los profesionales en el campo de la educación. Aquí también se debe reconocer los logros adquiridos por el docente a lo largo de su vida como profesional como es la capacitación, actualización, publicaciones y la experiencia que vaya adquiriendo en su trayectoria.

En consecuencia, el objetivo general del trabajo es indagar la transparencia y veracidad de los resultados del concurso de méritos y oposición, en el que participan aspirantes que buscan alcanzar un nombramiento o llenar una vacante en la carrera educativa pública dentro del Sistema Educativo del Ecuador.

Según el diccionario de la RAE (s.f) un concurso "es un procedimiento para seleccionar a quien haya de cubrir un puesto de trabajo mediante la realización de ciertas pruebas o exámenes". En este proceso los exámenes se realizan de diferente forma, con la finalidad de seleccionar al adecuado que ocupe una vacante. También concurso es una "forma de reclutamiento ordinario de 
los funcionarios, consistente en una selección y una clasificación de los candidatos, hechas por un jurado independiente, que se pronuncia sea con base en exámenes escritos u orales, sea por apreciación comparada de los título s universitarios" (Enciclopedia Jurídica, s.f).

El mérito es un valor o importancia resultado de las buenas acciones de una persona que lo hace digno de aprecio. Por el trabajo o el esfuerzo realizado es reconocido con ascenso, diplomas, medalla o alguna otra cosa que destaque los actos reconocidos. RAE, (s.f).

Según el diccionario de la RAE (s.f) la oposición "es un conjunto de pruebas selectivas en que los aspirantes a un puesto de trabajo, generalmente en la Administración pública, muestran su competencia, que es juzgada por un tribunal" En general el concurso de méritos y oposición según el Art. 183 del Reglamento a la LOSEP consiste en:

Art. 183.- Concurso de méritos y oposición. - Es el proceso orientado a seleccionar a las o los aspirantes que demuestren poseer las mejores competencias conforme a los requisitos del puesto, considerando los siguientes componentes: a) Mérito. - Es el análisis y verificación de las competencias documentadas presentadas por las y los aspirantes, conforme a lo establecido en la convocatoria; b) Oposición. - Es el análisis y verificación de las pruebas de conocimientos técnicos y psicométricas rendidas por el aspirante y la posterior entrevista. (Asamblea Constituyente., 2011).

Normas según Hans (2015) es expresar en el adjetivo "normal", "es lo que de hecho ocurre regularmente. En tanto que con ello se quiere significar también un deber ser, se presupone la validez de la norma que dice que lo que regularmente suele". Es el acto con que se ordena o prescribe algo, quiere que algo deba suceder.

Ganador "es el que gana, obtiene, triunfa, vence, logra, aventaja, acede y rebasa a alguien o algo por lo general a un pleito, concurso, juego, deporte, oposición, en un sueldo, remuneración y también de su voluntad" Santafé (2019).

Se entiende por apelación al acto por el que la parte que se considera perjudicada por una resolución acude al órgano superior jerárquico al que la dictó intentando su modificación. (Enciclopedia Jurídica, s.f).

Para Jaramillo (2016) la apelación en el recurso de apelación en la doctrina del Derecho Procesal "es el acto jurídico procesal de la parte agraviada o que ha sufrido un gravamen irreparable con la dictación de una resolución judicial por medio del cual solicita al Tribunal que la dictó que eleve el conocimiento del asunto al Tribunal superior jerárquico con el objeto de que este le enmiende con arreglo a derecho"

\section{Normativas legales que fundamenta el proceso de selección:}

El Estado tiene como objetivo de promover la igualdad real a favor de los titulares de derechos que se encuentren en situación de desigualdad, así la Constitución lo manifiesta en su Art. 11 Lit. 
2 "Todas las personas son iguales y gozaran de los mismos derechos, deberes y oportunidades" Asamblea Constituyente (2008) p.21. Tiene que adoptar medidas de acción afirmativa. Además, debe fortalecer las características materiales e inmateriales de la identidad observando que ninguna norma jurídica restrinja el contenido de los derechos y de las garantías constitucionales.

En la constitución del Ecuador se contemplan algunos artículos que hacen referencia al concurso de méritos y oposición. Es un procedimiento necesario que es realizado para que los docentes tengan la oportunidad de ingresen a trabajar en el sector público (Ministerio de Educación).

Art. 228.- El ingreso al servicio público, el ascenso y la promoción en la carrera administrativa se realizarán mediante concurso de méritos y oposición, en la forma que determine la ley, con excepción de las servidoras y servidores públicos de elección popular o de libre nombramiento y remoción. Su inobservancia provocará la destitución de la autoridad nominadora. (Asamblea Constituyente, 2008)

Art. 229.- "Serán servidoras o servidores públicos todas las personas que en cualquier forma o a cualquier título trabajen, presten servicios o ejerzan un cargo, función o dignidad dentro del sector público"p. 118.

Dentro del marco de la Ley Orgánica de Educación Intercultural (LOEI) en el capítulo segundo de los concursos de méritos y oposición para llenar vacantes de docentes públicos. Se toma en cuenta el Art. 97.- Vacantes. - "Las vacantes se producen cuando un docente cesa en sus funciones por renuncia, destitución, jubilación o fallecimiento, o cuando se crea una nueva partida presupuestaria a partir del desdoblamiento de partidas de docentes jubilados o mediante incrementos presupuestarios", así también la manifiesta la LOSEP en su artículo 66:

Art. 66.- De los puestos vacantes. - Para llenar los puestos vacantes se efectuará un concurso público de merecimientos y oposición. (Asamblea Nacional , 2010).

En este concurso participan docentes para ingresar a la Carrera pública y docentes mediante solicitud de cambio de institución, para ello en el Ate. 65 de la LOSEP "El ingreso a un puesto público será efectuado mediante concurso de merecimientos y oposición, que evalúe la idoneidad de los interesados y se garantice el libre acceso a los mismos" p. 41. En la educación intercultural bilingüe estas son llenadas por docentes que dominen el idioma natal al pueblo o nacionalidad a la que pertenecen. Con la finalidad de brindar una oportunidad de empleo a las personas que hablan idiomas ancestrales y conservar la lengua materna así manifiesta la LOEI Art. 94 Lit. f "En el caso de la educación intercultural bilingüe, el o la docente debe acreditar el dominio de un idioma ancestral". LOEI (2016) p. 58.

La convocatoria para llenar vacantes según el Art. 99 de la LOEI (2016) se lo realizará en un plazo no mayor a los 30 días este será publicada en los medios de comunicación pública de circulación nacional y en la página web del Ministerio de Educación. En el caso de no llenar el puesto en el tiempo determinado la autoridad nominadora será la responsable. 
Para obtener la calidad de legibles los participantes primero tendrán que inscribirse y registrar o actualizar sus datos en el Sistema de Información del Ministerio de Educación (SIME) en la sección denominada "Obtención de elegibilidad". Luego deben superar la prueba psicométrica y la prueba estandarizada de conocimientos específicos.

Las bases del concurso según el Art. 101 de la LOEI (2016) los candidatos rendirán pruebas de conocimientos generales y específicos de la materia, nivel y vacante. En caso de una institución intercultural bilingüe se tomara en cuenta en domino del idioma ancestral. Al finalizar los puntajes serán sumados (clase demostrativa, pruebas de conocimiento, méritos y bonificaciones).

A los candidatos finalistas o mejor puntuados se convoca a una clase demostrativa y entrevista en la instrucción educativa en la que este concursando. La autoridad máxima junto al gobierno escolar conformaran el jurado. Este no pueden participar familiares por parte del participante así lo señala el Reglamento a la LOSEP en su:

Art. 186.- Tribunales de méritos y oposición, y de apelaciones. - Para la ejecución de los concursos de méritos y oposición, las instituciones del Estado conformarán tribunales de méritos y oposición y de apelaciones, de conformidad con la normativa que emita el Ministerio de Relaciones Laborales (Asamblea Constituyente., 2011)

Al finalizar este proceso los resultado serán emitidos a la Autoridad Educativa Nacional. Así también lo señala el artículo 102 de la LOEI (2016):

Art. 102.- Calificación de méritos. - La autoridad nominadora respectiva de la Autoridad Educativa Nacional se encargará de calificar los méritos de los candidatos elegibles para llenar las vacantes, a través de sus respectivas unidades administrativas (...). Según el Art. 78 de la LOSEP las calificaciones son: excelente, muy bueno, satisfactorio, regular e insuficiente.

Los participantes gozan del derecho a la apelación. "Los participantes podrán solicitar la recalificación a sus expedientes y pruebas, dentro del término de cinco días contados a partir de la publicación de los resultados, bien sea por medios físicos, electrónicos o virtuales. La recalificación será resuelta y notificada en un término de treinta días improrrogables." La recalificación según la LOSEP en el ART.78 se realizará un tribunal integrado por tres servidores que no hayan intervenido en la calificación inicial.

Se considera elegibilidad preferente con puntaje adicional en el Art.103 (LOEI, 2016) a todos los postulantes que hayan laborado en zonas rurales por más de dos años, tengan su domicilio cerca del lugar donde exista una vacante, docentes mujeres jefas de hogar, candidatos elegibles con alguna discapacidad certificada. Así, también lo manifiesta y citamos en Art. 98 Traslados de planificación de partidas docentes Lit. a "Los que deban vivir cerca de un centro de salud por necesidad de atención médica especializada o por discapacidad propia, o de un familiar hasta el segundo grado de consanguinidad o primero de afinidad, y que dependa económicamente (...). Y en su Lit. d "Los que hayan laborado en zonas rurales" p. 60. 
En base al Art.104 de la LOEI los resultados del concurso serán publicados a través de la página web de la institución y la web del Ministerio de Relaciones Laborales. El candidato que obtenga la mejor calificación sumado a las pruebas, méritos, clase demostrativa y puntajes adicionales será acreedor al nombramiento para cubrir la vacante

Art. 17 del Reglamento General a la LOSEP (2011) Clases de nombramientos. - Los nombramientos extendidos para el ejercicio de un puesto en la función pública pueden ser: a) Permanentes; b) Provisionales; c) de libre nombramiento y remoción: Los expedidos a favor de personas que van a ocupar puestos de dirección política, estratégica o administrativa en las instituciones del Estado; y, d) De período fijo: Aquellos cuyos titulares son nombrados para ejercer un puesto en el servicio público por un período determinado por mandato legal.

En el caso de que este no se posesione el puesto o nombramiento será asignado al siguiente mejor puntuado. Así también lo establece el Reglamento General a la LOSEP en su Art. 187:

En el caso de que la ganadora o el ganador del concurso de Méritos y Oposición no aceptare el nombramiento, o no se presentare en la Institución para posesionarse del mismo, dentro del término de tres días a partir de la fecha de registro del nombramiento, el Tribunal de Méritos y Oposición declarará ganadora o ganador del concurso a la o el participante que haya obtenido el segundo mayor puntaje, y así sucesivamente, (...) (Asamblea Constituyente., 2011).

En el Art. 107 de la LOEI el proceso guarda el principio de transparencia y publicidad, mostrara los resultados parciales y finales del concurso. Por otro lado, en el Reglamento General a la LOSEP (2011) Art. 177 principios del subsistema señala en su Lit. b: “Transparencia. - La aplicación de métodos y procedimientos para seleccionar el talento humano competente, responderá a un tratamiento técnico, transparente, objetivo e imparcial para todos los aspirantes a desempeñar un puesto público" y en el Lit. f: "Difusión. - La difusión en la convocatoria y conocimiento, en las normas del concurso de méritos y oposición y su respectivo procedimiento y resultados" (Asamblea Constituyente., 2011) p. 50.

Los requisitos para los concursos de méritos y oposición son según el Art. 110 de la Ley Orgánica de Educación Intercultural Bilingüe son:

Art. 110.- Requisitos para los concursos públicos de méritos y oposición. - Los concursos públicos de méritos y oposición para ser directivo de una institución educativa pública incluyen los siguientes requisitos: a) Tener título profesional docente, o ser profesional de un área de interés para el sector educativo con título de post grado relacionado a educación; b) Aprobar las evaluaciones (...); c) Haber sido docente de aula al menos durante cinco años; d) Aprobar la prueba de selección para ser directivo, establecida por la Autoridad Educativa Nacional; y, e) Dominar un idioma ancestral en el caso de instituciones interculturales bilingües. (LOEI, 2016).

De acuerdo No MINEDUC-MINEDUC-2017-00065-A. Los actores educativos deben cumplir con la "Normativa para obtener la calidad de elegible y del concurso de méritos y oposición para llenar 
vacantes de docentes en el Magisterio Nacional" (Ministerio de Educación, 2017). Y cumplir con el proceso acorde al Acuerdo Ministerial Nro. MINEDUC-MINEDUC-2017-00065-A

\section{Metodología}

La investigación realizada fue bibliográfica, se recopilo información de fuentes confiables. Además, se hizo un análisis de las normativas legales vigentes para el proceso. Es de campo, participaron 10 docentes de diferentes Unidades Educativas del cantón Ambato, provincia de Tungurahua. La técnica y el instrumento aplicado fue creado por nuestro equipo de investigadores, las preguntas del cuestionario están enfocadas a indagar el proceso, la agilidad, la transparencia y la pertinencia en el concurso de méritos y oposición, previo a la obtención del nombramiento en el magisterio público. Este consta de 10 preguntas, 7 de ellas son pregunta abiertas porque necesitamos recolectar información de cómo se está dando en proceso y cuanto conocen del mismo; y 3 preguntas de seleccionar alternativas con un apartado para detallar la opción seleccionada. Esta permite determinar como el Mineduc realiza el concurso con los docentes en el país.

\section{Procedimiento}

El instrumento se validó mediante la aplicación de una prueba piloto a 2 docentes que están participando en el proceso. Luego fue aplicado dentro de las instituciones a los docentes de las diferentes áreas del conocimiento incluido cultura física, quienes llenaron el cuestionario en sus respectivos puesto de trabajo, llenar el cuestionario se tomó entre 10 a 20 minutos, se aseguró el completo anonimato y la participación fue voluntaria, con una aceptación de $100 \%$ de los encuestados.

Las preguntas aplicadas abordan indagar la transparencia y veracidad de los resultados del concurso de méritos y oposición, en el que participan aspirantes que buscan alcanzar un nombramiento o llenar una vacante en la carrera educativa publica dentro del Sistema Educativo del Ecuador

\section{Problema de investigación}

La investigación se centraliza en la siguiente pregunta: ¿Cuál es el proceso que siguen los aspirantes que buscan alcanzar un nombramiento o llenar una vacante en el Magisterio Nacional?

\section{Resultados}

Los docentes de las diferentes instituciones de la ciudad de Ambato

\section{¿Qué tiempo se tardó en conseguir su primer empleo en un establecimiento educativo?}

Gráfico 1. Tiempo para conseguir el primer empleo. 
Fuente: Encuesta aplicada a los aspirantes y ganadores del concurso.

Elaborado por: Autores.

\section{Análisis e Interpretación.}

De un total de 10 docentes, el 60\% tardaron más de tres años en ocupar un cargo docente, mientras que el $20 \%$ demoro dos años y el $20 \%$ un año.

Se deduce que la mayoría de la población tarda mucho tiempo en ocupar un puesto después de incorporase, existe mucha demanda de docentes y competencia profesional es por eso que no hay vacantes muy seguido, además la mayoría de las instituciones educativas piden como requisito tener experiencia. Ocupan un puesto después de dos años pero hasta el momento cruzan cursos de preparación, trabajan como reemplazo. Se tardan un año o menos porque fueron recomendados por amistades y anteriormente prepararon su carpeta profesional mediante cursos

Cuadro 4. Resultados del grupo focal docentes participantes del concurso de méritos y oposición

\begin{tabular}{ccc}
\hline Preguntas & Datos relevantes & $\begin{array}{c}\text { Criterios Predomi- } \\
\text { nantes }\end{array}$ \\
\hline
\end{tabular}

Si se cumple, pero hay que esperar mucho tiempo.

Tiene todos los procesos de acuerdo a la LOEI.

2. ¿De qué manera la normativa legal vigente se aplica el concurso de méritos y oposición? rente.
Cumple con lo establecido en la Ley vigente.

Se respeta el proceso divido en méritos y la oposición.

Es muy lento y comA mi parecer se aplica de manera transpa- plicado el proceso

Está sujeto a la ley.

Dependiendo el tiempo de trabajo y curso realizados.

Realizar todo el proceso de méritos y oposición.

Lo realizan de manera secuencial, pero es muy lento el proceso.

Es un poco complicado ya que se necesita no solo cumplir con la documentación correspondiente sino también presentarse a rendir pruebas que muestre el conocimiento que tenga. hasta llegar a la etapa final que es la clase demostrativa.

\section{Puntos de reflexión}

En la actualidad el proceso es transparente, se respeta lo establecido en la LOEI y su acuerdo ministerial para el concurso. El proceso es complicado debido a las pruebas que se aplican y a la documentación que toca presentar.

Transcurre de manera secuencial pero el lento hasta llegar a la etapa final que es la clase demostrativa. 
Respetando el proceso de las tres evalua-

ciones y la clase demostrativa

3. ¿Cuál es el proceso para la inscripción y participación en el concurso de méritos y oposición? y ¿cóm se realiza?

4. ¿En qué consiste y cuál es el proceso para adquirir la elegibilidad?
El aspirante al magisterio tiene que rendir dos pruebas para la elegibilidad, prueba de personalidad (idoneidad) y prueba de razonamiento lógico, verbal y matemático (elegibilidad). Una vez que tiene la elegibilidad debe esperar a las siguientes fases.

Registrarse en la pla-

taforma (SIME).

gina Web del Ministerio de Educación, se adjunta los méritos y a través de un cronograma se asiste a rendir las distintas pruebas. "Quiero ser Maestro"

Seguir en cronograma establecido.

Rendir las pruebas de La elegibilidad permite contipersonalidad y de las nuar las siguientes fases. Para de razonamiento ló- obtenerla debe rendir dos tipos gico, verbal y mate- de pruebas y obtener una punmático. tuación mínima de 7.

En la fase de méritos se sube al

Es la ubicación de las partidas vacantes y Informar el record sistema: títulos, certificados de la selección del circuito al que ese elegir. profesional (títulos).

En esta fase se debe registrar el título. $\quad$ Logros personales.

5. ¿En qué consiste la fase de méri- Consiste en la aprobación de pruebas y la tos y la fase de opo- clase demostrativa con más el $70 \%$ para pasición?

sar a la fase de oposición.
Validar los documentos de preparación.

Informar el récord profesional y en oposición aplicar la necesidad.

Ingresar los logros personales y experiencia. Ingresar las calificaciones de las pruebas y clase demostrativa.

Cursos o seminarios.

6. ¿Qué estudios posteriores está realizando o ha realizado?
Aprobar las pruebas

de conocimiento.

Aprobar la clase demostrativa. capacitación y publicaciones, es decir la hoja de vida.

En la oposición se rinde la prueba de conocimientos específicos de EGB y la clase demostrativa.
Doctorado.

Los cursos o seminarios que se Cursos o seminarios. hayan cruzado suman puntos a los méritos al igual que el título. Los docentes realizan actualización de conocimientos, se preparan para brindar una educación de calidad.

Elevan su grado de preparación no se quedan solo con el título de tercer nivel.

Para algunos aspirantes existe

No se aplica en este QSM6

7. ¿En qué consiste las bonificaciones adicionales sobre el puntaje obtenido y en qué situación se aplica?
Obtener una mejor desconocimiento de las bonificalificación. caciones y se les aplico en el QSM6.

Si la residencia está cercana a la institu- Mejora su puntuación final. ción.

Existe algunos aspectos para ser aplicados las bonificaciones.
La experiencia docente.

Obtención de la mejor calificación.

Por la residencia si está cercana a la institución.

\section{Educación Digital.}


Por crear artículos científicos.

(Didáctica, pedagogía, plan de clase, portafolio, LOEI).

8. Mencione 5 criterios de evaluación utilizados por parte del Mineduc durante la clase demostrativa.
(Practico, teórico, demostrativo, inductivo, motivación).

(Participación del estudiante, metodología

ERCA, objetivo, destrezas, material).

(Motivación, actividades iniciales, actividades de aprendizaje, actividades finales y evaluación, clima afectivo).
Evalúan el cumplimiento de objetivo o destrezas.

La motivación es un criterio fundamental en la clase demosgía, material didáctico, actividades a realizar y la motiva- Una metodología activa. ción).

El material utilizado despierta interés y participación de los estudiantes.

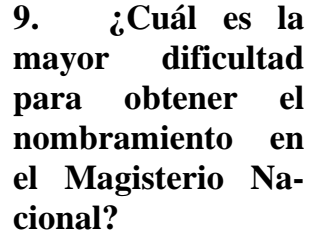

\section{Convocatoria limi- La convocatoria es limitada por tada por el Mineduc. \\ el Mineduc debido a que no existen vacantes muy seguido.}

10. ¿Cree usted que el proceso del concurso de méritos y oposición vigente es transpa- Es transparente puesto que se revisa en lírente, ágil y perti- nea todos los puntajes. nente?
Es transparente, pero no es ágil se tarda Es transparente.
mucho tiempo.

Se publica los puntajes.

Tarda mucho tiempo.
El proceso se realiza de forma justa y transparente sin perjudicar a nadie, los puntajes son publicados en la página oficial.

Tarda mucho tiempo debido al cronograma y las fases que debe seguir en aspirante.

Fuente: Encuesta aplicada a los aspirantes y ganadores del concurso.

Elaborado por: Autores

\section{Interpretación.}

En la ciudad de Ambato el proceso del concurso de méritos y oposición por el que deben pasar los profesionales en educación aspirantes a un nombramiento, es transparente y cumple a cabalidad con lo establecido en la Ley Orgánica de Educación Intercultural (LOEI) en el capítulo segundo de los concursos de méritos y oposición para llenar vacantes de docentes públicos y su Acuerdo No MINEDUC-MINEDUC-2017-00065-A. "Normativa para obtener la calidad de elegible y del concurso de méritos y oposición para llenar vacantes de docentes en el Magisterio Nacional", desde su inicio hasta su fase final la clase demostrativa. Es transparente por todos los resultados son publicados en la página oficial del Mineduc.

Para participar en este proceso de selección primero se debe registrar por primera vez en el Sistema de Información del Ministerio de Educación (SIME), completar todos los datos informativos y seguir en cronograma establecido. Luego adquirir la elegibilidad esta facultad le permitirá al aspirante continuar a las siguientes fases, se debe rendir las pruebas psicométricas y de conocimientos específicos, este debe ser aprobada con una puntuación mínima del $70 \%$. 
Lo que concierne a la fase de méritos el $100 \%$ de los aspirantes manifiestan que consiste en el récord o currículo profesional docente, este debe ser subido a la plataforma SIME. La fase de oposición son las pruebas de conocimientos y finalmente la clase demostrativa. Los docentes en la actualidad el $100 \%$ se preocupan por su perfil profesional, realizan cursos de actualización, además continúan sus estudios en busca de otro título profesional. La finalidad es mejorar la calidad educativa y no solo obtener un mayor puntaje en sus méritos.

La mayor dificultad para obtener un nombramiento en el Magisterio Nacional es la convocatoria limitada por el Mineduc y las pruebas que se aplican durante todo el proceso. Este es pertinente porque permite seleccionar a los mejores profesionales, es transparente e igualitario, pero demora mucho tiempo culminar el procedo debido al cronograma establecido y las fases que se deben cumplir.

\section{Discusión.}

El ministerio de Educación cumple a cabalidad el proceso de selección, pero no todos los participantes conocen la normativa legal para este proceso. Las bonificaciones benefician a los aspirantes en su puntuación final, algunos manifiestan que desconocen los casos en los que se aplica y que en el QSM6 no se aplicó esto. Además, no tienen claro los parámetros para realizar una clase demostrativa, en este aparto los que más se evalúa es la motivación, el cumplimiento de los objetivos y la metodología a emplear.

Los resultados conllevan un proceso eficaz en donde existe la transparencia, motiva a los aspirantes a participar en el proceso de selección, muestran toca su capacidad y potencial. Existe un problema en la eficiencia, el tiempo que se toma en cumplir todo el proceso causa desinterés en los participantes, a pesar de esta dificultan continúan hasta el final.

La principal limitación para el ingreso al sistema educativo es la convocatoria, debido a que no existe vacantes disponibles. El bajo puntaje en los méritos por falta de cursos, la falta de experiencia laboral es decir trabajan a contrato sin aportación al IESS, publicaciones y en la oposición la preparación de la clase demostrativa.

Las fortalezas para realizar el estudio son conocer la ley y su instructivo para el concurso, colaboración de los docentes, predisponían del tiempo, medios de transporte y el uso de las TICS. Dentro de las debilidades no se facilita el acceso a las instituciones educativas y los participantes desconocen la normativa legal.

Se ha logrado el objetivo propuesto que es indagar la transparencia y veracidad de los resultados del concurso de méritos y oposición, en el que participan aspirantes que buscan alcanzar un nombramiento o llenar una vacante en la carrera educativa pública dentro del Sistema Educativo del Ecuador. Se logró mediante la investigación bibliográfica y de campo, la aplicación de una técnica que permitió conocer la transparencia del proceso y el criterio de cada uno de los participantes. 


\section{Conclusiones.}

- En conclusión, el trabajo que realiza el Mineduc por medio de la plataforma del Sistema de información del Ministerio de Educación (SIME) convoca a los profesionales en educación a participar en los concursos de méritos y oposición, actualmente llamadas las pruebas "SER MAESTRO". Este se lo realiza de forma igualitaria y transparente con la finalidad de los perjudicar a los docentes, por el contrario, busca reconocer su potencial y brindar la oportunidad de alcanzar un nombramiento en Magisterio.

- Se evidencia que los aspirantes que participaron en este proceso conocen la normativa legal, además las fases que se debe seguir para alcanzar el nombramiento. Están conscientes del esfuerzo que deben realizar, muestran un espirito de superación y el compromiso al desarrollo de la educación en el país.

- De acuerdo a los resultados, el tiempo que un docente tarda en desempeñarse en su profesión es aceptable en algunos casos demora mucho porque la demanda profesional incrementa a medida que pasa el tiempo, se saturan las vacantes.

- El tiempo que un aspirante debe esperar para lograr ingresar al magisterio y ocupar un nombramiento es tedioso, debido a la limitada convocatoria de vacantes; muy extenso, debido a que debe seguir un proceso sistemático por fases y cada una debe ser superada de acuerdo al cronograma establecido por el Ministerio de Educación.

- En las bonificaciones que son brindadas al aspirante, sumaran puntos extras en caso de ser aplicados. Los resultados enmaran que los participantes desconocen de este beneficio y cuando no más se aplica, creen que solo se aplica a las personas con discapacidad y muchas de las veces no son tomadas en cuenta durante en proceso.

\section{Referencias bibliográficas.}

Asamblea Constituyente. (2008). Constitución de la Republíca del Ecuador. Quito. Obtenido de https://www.oas.org/juridico/mla/sp/ecu/sp_ecu-int-text-const.pdf

Sanza, C. X. (2018). Los Contratos Ocasionales y/o Nombramientos Provisionales en las Instituciones Públicas Vulneran el Derecho Constitucional de Igualdad en los Concursos de Méritos y Oposición. Obtenido de http://dspace.unl.edu.ec/jspui/bitstream/123456789/21456/1/Carlos\%20Xavier\%20Barrig a\%20Asanza.pdf

Cari, J. M. (2016). El concurso público de méritos para el ingreso a la administración pública. Obtenido de http://www.la-razon.com/la_gaceta_juridica/concurso-publico-meritosadministracion-publica_0_2488551149.html

Costa Jaramillo, M. L. (2012). Concurso de Meritos y Oposiciones. Obtenido de http://dspace.utpl.edu.ec/bitstream/123456789/5699/1/Costa\%20Jaramillo\%20Maria\%20 Lorena.pdf 
LOEI. (2016). Ley Orgánica de Educación Intercultural Bilingue. Quito. Obtenido de Asamblea Nacional: https://educacion.gob.ec/wpcontent/uploads/downloads/2017/02/Ley_Organica_de_Educacion_Intercultural_LOEI_c odificado.pdf

Ministerio de Educación. (2017). MINEDUC-MINEDUC-2017-00065-A. Quito.

Estrada Guerrero, R., Lemus Torres, D., Mendoza Anaya, D., \& Rodríguez Lugo, V. (2010). Hidrogeles poliméricos potencialmente aplicables en Agricultura. Revista Iberoamericana de Polímeros, 12(2), 76-87.

Asamblea Nacional . (2010). LEY ORGANICA DE SERVICIO PUBLICO. Quito. Obtenido de https://educacion.gob.ec/wpcontent/uploads/downloads/2017/02/Ley_Organica_de_Educacion_Intercultural_LOEI_c odificado.pdf

Costa Jaramillo, M. L. (2012). Concurso de Méritos y Oposiciones. Obtenido de http://dspace.utpl.edu.ec/bitstream/123456789/5699/1/Costa\%20Jaramillo\%20Maria\%20 Lorena.pdf

Enciclopedia Jurídica. (s.f). definición de concurso. Edición 2020. Obtenido de Enciclopedia Jurídica: http://www.enciclopedia-juridica.com/d/concurso/concurso.htm

Asamblea Constituyente. (2008). Constitución de la República del Ecuador. Quito. Obtenido de https://www.oas.org/juridico/mla/sp/ecu/sp_ecu-int-text-const.pdf

Asamblea Constituyente. (2011). REGLAMENTO GENERAL A LA LEY ORGANICA DEL SERVICIO PUBLICO. Quito: Ultima modificación: 28-sep.-2018.

Asamblea Nacional . (2010). LEY ORGANICA DE SERVICIO PUBLICO. Quito. Obtenido de https://educacion.gob.ec/wp-

content/uploads/downloads/2017/02/Ley_Organica_de_Educacion_Intercultural_LOEI_c odificado.pdf

Asanza, C. X. (2018). Los Contratos Ocasionales y/o Nombramientos Provisionales en las Instituciones Públicas Vulneran el Derecho Constitucional de Igualdad en los Concursos de Méritos y Oposición. Obtenido de http://dspace.unl.edu.ec/jspui/bitstream/123456789/21456/1/Carlos\%20Xavier\%20Barrig a\%20Asanza.pdf

Cari, J. M. (2016). El concurso público de méritos para el ingreso a la administración pública. Obtenido de http://www.la-razon.com/la_gaceta_juridica/concurso-publico-meritosadministracion-publica_0_2488551149.html 
Costa Jaramillo, M. L. (2012). Concurso de Méritos y Oposiciones. Obtenido de http://dspace.utpl.edu.ec/bitstream/123456789/5699/1/Costa\%20Jaramillo\%20Maria\%20 Lorena.pdf

Enciclopedia Jurídica. (s.f). definición de concurso. Edición 2020. Obtenido de Enciclopedia Jurídica: http://www.enciclopedia-juridica.com/d/concurso/concurso.htm

García Chávez, J. R. (2009). CONTROL DE CONCURSOS DE MÉRITOS Y OPOSICIÓN EN JURISPRUDENCIA.

Obtenido

de https://books.google.es/books?hl=es\&lr=\&id=UC16EFUHy9UC\&oi=fnd\&pg=PA7\&dq= concurso+de+méritos+y+oposiciones\&ots=m4FUW_1aIZ\&sig=qZz6FDwmHkeiq2k0i1 YtjQmpq4\#v=onepage \&q=concurso\%20de\%20meritos\%20y\%20oposicion $\mathrm{es} \& \mathrm{f}=\mathrm{false}$

LOEI. (2016). Ley Orgánica de Educación Intercultural Bilingüe. Quito. Obtenido de Asamblea Nacional: https://educacion.gob.ec/wpcontent/uploads/downloads/2017/02/Ley_Organica_de_Educacion_Intercultural_LOEI_c odificado.pdf

Ministerio de Educación. (2017). MINEDUC-MINEDUC-2017-00065-A. Quito.

Moscoso, J., \& Espín, S. (2011). ANÁLISIS ESTRUCTURAL DE LOS CONCURSOS DE MÉRITOS Y OPOSICIÓN. Obtenido de http://repositorio.puce.edu.ec/bitstream/handle/22000/12708/TESIS\%20JOSE\%20ESPIN .pdf?sequence $=1$

Samaniego Dumas, M. P. (2017). APLICACIÓN DE LA MEDIDA DE ACCIÓN AFIRMATIVA, AUTO DEFINICIÓN ÉTNICA. Obtenido de http://dspace.uniandes.edu.ec/bitstream/123456789/7619/1/PIUAMCO009-2018.pdf

Sarango, $\quad$ A. $\quad$ T. (2016). Obtenido de http://dspace.unl.edu.ec/jspui/bitstream/123456789/17310/1/Andrea\%20Tatiana\%20Quiz hpe\%20Sarango.pdf

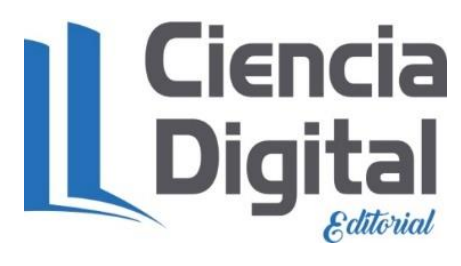




\section{PARA CITAR EL ARTÍCULO INDEXADO.}

Velasco Almachi, L. D., Ortega Araus, E. del R. O. A., Yungán Yungán, R. Y. Y., \& Tenelema Toapanta, V. J. (2020). Cumplimiento de las normas legales en el concurso de méritos y oposición del sistema educativo ecuatoriano. Ciencia Digital, 4(2), 102-119. https://doi.org/10.33262/cienciadigital.v4i2.1212

\section{¿Ciencia}

El artículo que se publica es de exclusiva responsabilidad de los autores y no necesariamente reflejan el pensamiento de la Revista Ciencia Digital.

El artículo queda en propiedad de la revista y, por tanto, su publicación parcial y/o total en otro medio tiene que ser autorizado por el director de la Revista Ciencia Digital.
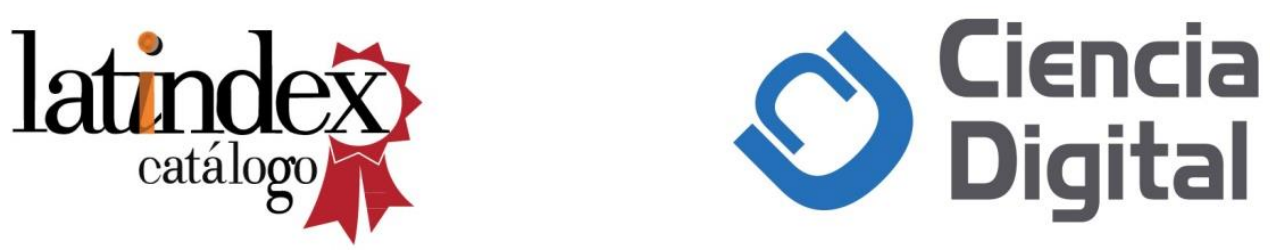